\title{
Modular Verification of Recursive Programs
}

\author{
Krzysztof R. Apt ${ }^{1,2}$, Frank S. de Boer ${ }^{1,3}$, and Ernst-Rüdiger Olderog ${ }^{4}$ \\ 1 Centre for Mathematics and Computer Science (CWI), Amsterdam, The Netherlands \\ 2 University of Amsterdam, Institute of Language, Logic and Computation, Amsterdam \\ ${ }^{3}$ Leiden Institute of Advanced Computer Science, University of Leiden, The Netherlands \\ ${ }^{4}$ Department of Computing Science, University of Oldenburg, Germany
}

\begin{abstract}
We argue that verification of recursive programs by means of the assertional method of C.A.R. Hoare can be conceptually simplified using a modular reasoning. In this approach some properties of the program are established first and subsequently used to establish other program properties. We illustrate this approach by providing a modular correctness proof of the Quicksort program.
\end{abstract}

\section{Introduction}

Program verification by means of the assertional method of Hoare (so-called Hoare's logic) is by now well-understood. One of its drawbacks is that it calls for a tedious manipulation of assertions, which is error prone. The support offered by the available by interactive proof checkers, such as PVS (Prototype Verification System), see [15], is very limited.

One way to reduce the complexity of an assertional correctness proof is by organizing it into a series of simpler proofs. For example, to prove $\{p\} S\left\{q_{1} \wedge q_{2}\right\}$ we could establish $\{p\} S\left\{q_{1}\right\}$ and $\{p\} S\left\{q_{2}\right\}$ separately (and independently). Such an obvious approach is clearly of very limited use.

In this paper we propose a different approach that is appropriate for recursive programs. In this approach a simpler property, say $\left\{p_{1}\right\} S\left\{q_{1}\right\}$, is established first and then used in the proof of another property, say $\left\{p_{2}\right\} S\left\{q_{2}\right\}$. This allows us to establish $\left\{p_{1} \wedge p_{2}\right\} S\left\{q_{1} \wedge q_{2}\right\}$ in a modular way. It is obvious how to generalize this approach to an arbitrary sequence of program properties for which the earlier properties are used in the proofs of the latter ones. So, in contrast to the simplistic approach mentioned above, the proofs of the program properties are not independent but are arranged instead into an acyclic directed graph.

We illustrate this approach by providing a modular correctness proof of the Quicksort program due to [10]. This yields a correctness proof that is better structured and conceptually easier to understand than the original one, given in [7]. A minor point is that we use different proof rules concerning procedure calls and also provide an assertional proof of termination of the program, a property not considered in [7]. (It should be noted that termination of recursive procedures with parameters within the framework of the assertional method was considered only in the eighties, see, e.g., [2]. In these proofs some subtleties arise that necessitate a careful exposition, see [1].)

We should mention here two other references concerning formal verification of the Quicksort program. In [6] the proof of Quicksort is certified using the interactive 
theorem prover Coq, while in [13] a correctness proof of a non-recursive version of Quicksort is given.

The paper is organized as follows. In the next section we introduce a small programming language that involves recursive procedures with parameters called by value and discuss its operational semantics. Then, in Section 3 we introduce a proof system for proving partial and total correctness of these programs. The presentation in these two sections is pretty standard except for the treatment of the call-by-value parameter mechanism that avoids the use of substitution.

Next, in Section 4 we discuss how the correctness proofs, both of partial and of total correctness, can be structured in a modular way. In Section 5 we illustrate this approach by proving correctness of the Quicksort program while in Section 6 we discuss related work and draw some conclusions. Finally, in the appendix we list the used axioms and proof rules concerned with non-recursive programs. The soundness of the considered proof systems is rigorously established in [3] using the operational semantics of [16 17].

\section{A Small Programming Language}

\section{Syntax}

We use simple variables and array variables. Simple variables are of a basic type (for example integer or Boolean), while array variables are of a higher type (for example integer $\times$ Boolean $\rightarrow$ integer). A subscripted variable derived from an array variable $a$ of type $T_{1} \times \ldots \times T_{n} \rightarrow T$ is an expression of the form $a\left[t_{1}, \ldots, t_{n}\right]$, where each expression $t_{i}$ is of type $T_{i}$.

In this section we introduce a class of recursive programs as an extension of the class of while programs which are generated by the following grammar:

$$
S::=\operatorname{skip}|u:=t| \bar{x}:=\bar{t}\left|S_{1} ; S_{2}\right| \text { if } B \text { then } S_{1} \text { else } S_{2} \text { fi } \mid \text { while } B \text { do } S_{1} \text { od, }
$$

where $S$ stands for a typical statement or program, $u$ for a simple or subscripted variable, $t$ for an expression (of the same type as $u$ ), and $B$ for a Boolean expression. Further, $\bar{x}:=\bar{t}$ is a parallel assignment, with $\bar{x}=x_{1}, \ldots, x_{n}$ a non-empty list of distinct simple variables and $\bar{t}=t_{1}, \ldots, t_{n}$ a list of expressions of the corresponding types. The parallel assignment plays a crucial role in our modelling of the parameter passing. We do not discuss the types and only assume that the set of basic types includes at least the types integer and Boolean. As an abbreviation we introduce if $B$ then $S$ fi $\equiv$ if $B$ then $S$ else skip fi.

Given an expression $t$, we denote by $\operatorname{var}(t)$ the set of all simple and array variables that appear in $t$. Analogously, given a program $S$, we denote by $\operatorname{var}(S)$ the set of all simple and array variables that appear in $S$, and by change $(S)$ the set of all simple and array variables that can be modified by $S$, i.e., the set of variables that appear on the left-hand side of an assignment in $S$.

We arrive at recursive programs by adding recursive procedures with call-by-value parameters. To distinguish between local and global variables, we first introduce a block statement by the grammar rule

$$
S::=\text { begin local } \bar{x}:=\bar{t} ; S_{1} \text { end. }
$$


A block statement introduces a non-empty sequence $\bar{x}$ of simple local variables, all of which are explicitly initialized by means of a parallel assignment $\bar{x}:=\bar{t}$, and provides an explicit scope for these simple local variables. The precise explanation of a scope is more complicated because the block statements can be nested.

Assuming $\bar{x}=x_{1}, \ldots, x_{k}$ and $\bar{t}=t_{1}, \ldots, t_{k}$, each occurrence of a local variable $x_{i}$ within the statement $S_{1}$ and not within another block statement that is a subprogram of $S_{1}$ refers to the same variable. Each such variable $x_{i}$ is initialized to the expression $t_{i}$ by means of the parallel assignment $\bar{x}:=\bar{t}$. Further, given a statement $S^{\prime}$ such that begin local $\bar{x}:=\bar{t} ; S_{1}$ end is a subprogram of $S^{\prime}$, all occurrences of $x_{i}$ in $S^{\prime}$ outside this block statement refer to some other variable(s).

Procedure calls with parameters are introduced by the grammar rule

$$
S::=P\left(t_{1}, \ldots, t_{n}\right),
$$

where $P$ is a procedure identifier and $t_{1}, \ldots, t_{n}$, with $n \geq 0$, are expressions called actual parameters. The statement $P\left(t_{1}, \ldots, t_{n}\right)$ is called a procedure call. The resulting class of programs is then called recursive programs.

Procedures are defined by declarations of the form

$$
P\left(u_{1}, \ldots, u_{n}\right):: S,
$$

where $u_{1}, \ldots, u_{n}$ are distinct simple variables, called formal parameters of the procedure $P$ and $S$ is the body of the procedure $P$.

We assume a given set of procedure declarations $D$ such that each procedure that appears in $D$ has a unique declaration in $D$. When considering recursive programs we assume that all procedures whose calls appear in the considered recursive programs are declared in $D$. Additionally, we assume that the procedure calls are well-typed, which means that the numbers of formal and actual parameters agree and that for each parameter position the types of the corresponding actual and formal parameters coincide.

Given a recursive program $S$, we call a variable $x_{i}$ local if it appears within a subprogram of $D$ or $S$ of the form begin local $\bar{x}:=\bar{t} ; S_{1}$ end with $\bar{x}=x_{1}, \ldots, x_{k}$, and global otherwise.

To avoid possible name clashes between local and global variables we assume that given a set of procedure declarations $D$ and a recursive program $S$, no local variable of $S$ occurs in $D$. So given the procedure declaration

$$
P:: \text { if } x=1 \text { then } b:=\text { true else } b:=\text { false fi }
$$

the program

$$
S \equiv \operatorname{begin~local~} x:=1 ; P \text { end }
$$

is not allowed. If it were, the semantics we are about to introduce would allow us to conclude that $\{x=0\} S\{b\}$ holds. However, the customary semantics of the programs in the presence of procedures prescribes that in this case $\{x=0\} S\{\neg b\}$ should hold, as the meaning of a program should not depend on the choice of the names of its local variables. (This is a consequence of the so-called static scope of the variables that we assume here.) 
This problem is trivially solved by just renaming the 'offensive' local variables to avoid name clashes, so by considering here the program begin local $y:=1 ; P$ end instead of $S$. Once we limit ourselves to recursive programs no local variable of which occurs in the considered set of procedure declarations, the semantics we introduce ensures that the names of local variables indeed do not matter. More precisely, the programs that only differ in the choice of the names of local variables and obey the above syntactic restriction have then identical meaning. In what follows, when considering a recursive program $S$ in the context of a set of procedure declarations $D$ we always implicitly assume that the above syntactic restriction is satisfied.

The local and global variables play an analogous role to the bound and free variables in first-order formulas or in $\lambda$-terms. In fact, the above syntactic restriction corresponds to the 'Variable Convention' of [4, page 26] according to which "all bound variables are chosen to be different from the free variables."

Note that the above definition of programs puts no restrictions on the actual parameters in procedure calls; in particular they can be formal parameters or global variables.

\section{Semantics}

For recursive programs we use a structural operational semantics in the sense of Plotkin [17]. As usual, it is defined in terms of transitions between configurations. A configuration $C$ is a pair $\langle S, \sigma>$ consisting a statement $S$ that is to be executed and a state $\sigma$ that assigns a value to each variable (including local variables). A transition is written as a step $C \rightarrow C^{\prime}$ between configurations. To express termination we use the empty statement $E$; a configuration $\langle E, \sigma\rangle$ denotes termination in the state $\sigma$.

Transitions are specified by the transition axioms and rules which are defined in the context of a set $D$ of procedure declarations. The only transition axioms that are somewhat non-standard are the ones that deal with the block statement and the procedure calls, in that they avoid the use of substitution thanks to the use of parallel assignment:

$<$ begin local $\bar{x}:=\bar{t} ; S$ end, $\sigma>\rightarrow<\bar{x}:=\bar{t} ; S ; \bar{x}:=\sigma(\bar{x}), \sigma>$,

$<P(\bar{t}) ; R, \sigma>\rightarrow<$ begin local $\bar{u}:=\bar{t} ; S$ end $R, \sigma>$,

where $P(\bar{u}):: S \in D$.

The first axiom ensures that the local variables are initialized as prescribed by the parallel assignment and that upon termination the global variables whose names coincide with the local variables are restored to their initial values, held at the beginning of the block statement. This is a way of implicitly modeling a stack discipline for (nested) blocks. So the use of the block statement in the second transition axiom ensures that prior to the execution of the procedure body the formal parameters are simultaneously instantiated to the actual parameters and that upon termination of a procedure call the formal parameters are restored to their initial values. Additionally, the block statement limits the scope of the formal parameters so that they are not accessible upon termination of the procedure call. So the second transition axiom describes the call-by-value parameter mechanism. 
Based on the transition relation $\rightarrow$ we consider two variants of input/output semantics for recursive programs $S$ refering to the set $\Sigma$ of states $\sigma, \tau$. The partial correctness semantics is a mapping $\mathcal{M} \llbracket S \rrbracket: \Sigma \rightarrow \mathcal{P}(\Sigma)$ defined by

$$
\mathcal{M} \llbracket S \rrbracket(\sigma)=\left\{\tau \mid<S, \sigma>\rightarrow^{*}<E, \tau>\right\} .
$$

The total correctness semantics is a mapping $\mathcal{M}_{\text {tot }} \llbracket S \rrbracket: \Sigma \rightarrow \mathcal{P}(\Sigma \cup\{\perp\})$ defined by

$$
\mathcal{M}_{\text {tot }} \llbracket S \rrbracket(\sigma)=\mathcal{M} \llbracket S \rrbracket(\sigma) \cup\{\perp \mid S \text { can diverge from } \sigma\} .
$$

Here $\perp$ is an error state signalling divergence, i.e., an infinite sequence of transitions starting in the configuration $\langle S, \sigma\rangle$.

\section{Proof Systems for Partial and Total Correctness}

Program correctness is expressed by correctness formulas of the form $\{p\} S\{q\}$, where $S$ is a program and $p$ and $q$ are assertions. The assertion $p$ is the precondition of the correctness formula and $q$ is the postcondition. A correctness formula $\{p\} S\{q\}$ is true in the sense of partial correctness if every terminating computation of $S$ that starts in a state satisfying $p$ terminates in a state satisfying $q$. And $\{p\} S\{q\}$ is true in the sense of total correctness if every computation of $S$ that starts in a state satisfying $p$ terminates and its final state satisfies $q$. Thus in the case of partial correctness, diverging computations of $S$ are not taken into account.

Using the semantics $\mathcal{M}$ and $\mathcal{M}_{t o t}$, we formalize these two interpretations of correctness formulas uniformly as set theoretic inclusions as follows (cf. [3]). For an assertion $p$ let $\llbracket p \rrbracket$ denote the set of states satisfying $p$. Then we define:

(i) The correctness formula $\{p\} S\{q\}$ is true in the sense of partial correctness, abbreviated by $\models\{p\} S\{q\}$, if $\mathcal{M} \llbracket S \rrbracket(\llbracket p \rrbracket) \subseteq \llbracket q \rrbracket$.

(ii) The correctness formula $\{p\} S\{q\}$ is true in the sense of total correctness, abbreviated by $\models_{t o t}\{p\} S\{q\}$, if $\mathcal{M}_{\text {tot }} \llbracket S \rrbracket(\llbracket p \rrbracket) \subseteq \llbracket q \rrbracket$.

Since by definition $\perp \notin \llbracket q \rrbracket$, part (ii) indeed formalizes the above intuition about total correctness.

\section{Partial Correctness}

Partial correctness of while programs is proven using the customary proof system $P D$ consisting of the group of axioms and rules 17 shown in the appendix. Consider now partial correctness of recursive programs. First, we introduce the following rule that deals with the block statement.

\section{BLOCK}

$$
\frac{\{p\} \bar{x}:=\bar{t} ; S\{q\}}{\{p\} \text { begin local } \bar{x}:=\bar{t} ; S \text { end }\{q\}}
$$

where $\operatorname{var}(\bar{x}) \cap \operatorname{free}(q)=\emptyset$. 
By free $(q)$ we denote here the set of all free simple and array variables that have a free occurrence in the assertion $q$.

The main issue is how to deal with the procedure calls. To this end, we want to adjust the proofs of 'generic' procedure calls to arbitrary ones. The definition of a generic call and the conditions for the correctness of such an adjustment process refer to the assumed set of procedure declarations $D$. By a generic call of a procedure $P$ we mean a call of the form $P(\bar{x})$, where $\bar{x}$ is a sequence of fresh (w.r.t. $D$ ) variables.

First, we extend the definition of change $(S)$ to recursive programs and sets of procedure declarations as follows:

$$
\begin{aligned}
& \text { change }(\text { begin local } \bar{x}:=\bar{t} ; S \text { end })=\text { change }(S) \backslash\{\bar{x}\}, \\
& \text { change }(P(\bar{u}):: S)=\text { change }(S) \backslash\{\bar{u}\}, \\
& \text { change }(\{P(\bar{u}):: S\} \cup D)=\text { change }(P(\bar{u}):: S) \cup \text { change }(D), \\
& \text { change }(P(\bar{t}))=\emptyset .
\end{aligned}
$$

The adjustment of the generic procedure calls is then taken care of by the following proof rule that refers to the set of procedure declarations $D$ :

\section{INSTANTIATION}

$$
\frac{\{p\} P(\bar{x})\{q\}}{\{p[\bar{x}:=\bar{t}]\} P(\bar{t})\{q[\bar{x}:=\bar{t}]\}}
$$

where $\operatorname{var}(\bar{x}) \cap \operatorname{var}(D)=\operatorname{var}(\bar{t}) \cap \operatorname{change}(D)=\emptyset$ and $P(\bar{u}):: S \in D$ for some $S$.

In the following rule for recursive procedures with parameters we use the provability symbol $\vdash$ to refer to the proof system $P D$ augmented with the auxiliary axiom and rules A1 A6 defined in the appendix and the above two proof rules.

\section{RECURSION}

$$
\begin{aligned}
& \left\{p_{1}\right\} P_{1}\left(\bar{x}_{1}\right)\left\{q_{1}\right\}, \ldots,\left\{p_{n}\right\} P_{n}\left(\bar{x}_{n}\right)\left\{q_{n}\right\} \vdash\{p\} S\{q\}, \\
& \left\{p_{1}\right\} P_{1}\left(\bar{x}_{1}\right)\left\{q_{1}\right\}, \ldots,\left\{p_{n}\right\} P_{n}\left(\bar{x}_{n}\right)\left\{q_{n}\right\} \vdash \\
& \quad\left\{p_{i}\right\} \text { begin local } \bar{u}_{i}:=\bar{x}_{i} ; S_{i} \text { end }\left\{q_{i}\right\}, i \in\{1, \ldots, n\} \\
& \hline
\end{aligned}
$$

$\{p\} S\{q\}$

where $D=P_{1}\left(\bar{u}_{1}\right):: S_{1}, \ldots, P_{n}\left(\bar{u}_{n}\right):: S_{n}$ and $\operatorname{var}\left(\bar{x}_{i}\right) \cap \operatorname{var}(D)=\emptyset$ for $i \in$ $\{1, \ldots, n\}$.

The intuition behind this rule is as follows. Say that a program $S$ is $(p, q)$-correct if $\{p\} S\{q\}$ holds in the sense of partial correctness. The second premise of the rule states that we can establish from the assumption of the $\left(p_{i}, q_{i}\right)$-correctness of the 'generic' procedure calls $P_{i}\left(\bar{x}_{i}\right)$ for $i \in\{1, \ldots, n\}$, the $\left(p_{i}, q_{i}\right)$-correctness of the procedure bodies $S_{i}$ for $i \in\{1, \ldots, n\}$, which are adjusted as in the transition axiom that deals with the procedure calls. Then we can prove the $\left(p_{i}, q_{i}\right)$-correctness of the procedure calls $P_{i}\left(\bar{x}_{i}\right)$ unconditionally, and thanks to the first premise establish the $(p, q)$-correctness of the recursive program $S$.

To prove partial correctness of recursive programs with parameters we use the proof system $P R$ that is obtained by extending the proof system $P D$ by the block rule, the instantiation rule, the recursion rule, and the auxiliary axiom and rules A1, A6. 
Note that when we deal only with one recursive procedure and use the procedure call as the considered recursive program, this rule simplifies to

$$
\frac{\{p\} P(\bar{x})\{q\} \vdash\{p\} \text { begin local } \bar{u}:=\bar{x} ; S \text { end }\{q\}}{\{p\} P(\bar{x})\{q\}}
$$

where $D=P(\bar{u}):: S$ and $\operatorname{var}(\bar{x}) \cap \operatorname{var}(D)=\emptyset$.

\section{Total Correctness}

Total correctness of while programs is proven using the proof system $T D$ consisting of the group of axioms and rules $1,5,7$ and 8 shown in the appendix. For total correctness of recursive programs we need a modification of the recursion rule. The provability symbol $\vdash$ refers now to the proof system $T D$ augmented with the auxiliary rules A2 A6 the block rule and the instantiation rule. The proof rule is a minor variation of a rule originally proposed in [1] and has the following form:

\section{RECURSION II}

$$
\begin{aligned}
& \left\{p_{1}\right\} P_{1}\left(\bar{x}_{1}\right)\left\{q_{1}\right\}, \ldots,\left\{p_{n}\right\} P_{n}\left(\bar{x}_{n}\right)\left\{q_{n}\right\} \vdash\{p\} S\{q\} \\
& \left\{p_{1} \wedge t<z\right\} P_{1}\left(\bar{x}_{1}\right)\left\{q_{1}\right\}, \ldots,\left\{p_{n} \wedge t<z\right\} P_{n}\left(\bar{x}_{n}\right)\left\{q_{n}\right\} \vdash \\
& \quad\left\{p_{i} \wedge t=z\right\} \text { begin local } \bar{u}_{i}:=\bar{x}_{i} ; S_{i} \text { end }\left\{q_{i}\right\}, i \in\{1, \ldots, n\} \\
& \hline\{p\} S\{q\}
\end{aligned}
$$

where $D=P_{1}\left(\bar{u}_{1}\right):: S_{1}, \ldots, P_{n}\left(\bar{u}_{n}\right):: S_{n}, \operatorname{var}\left(\bar{x}_{i}\right) \cap \operatorname{var}(D)=\emptyset$ for $i \in\{1, \ldots, n\}$, and $z$ is an integer variable that does not occur in $p_{i}, t, q_{i}$ and $S_{i}$ for $i \in\{1, \ldots, n\}$ and is treated in the proofs as a constant, which means that in these proofs neither the $\exists$-introduction rule $\mathrm{A} 4$ nor the substitution rule $\mathrm{A} 6$ defined in the appendix is applied to $z$.

To prove total correctness of recursive programs with parameters we use the proof system $T R$ that is obtained by extending the proof system $T D$ by the block rule, the instantiation rule, the recursion rule II, and the auxiliary rules $\mathrm{A} 2 \mathrm{~A} 6$.

As before, in the case of one recursive procedure this rule can be simplified to

$$
\begin{aligned}
& \{p \wedge t<z\} P(\bar{x})\{q\} \vdash\{p \wedge t=z\} \text { begin local } \bar{u}:=\bar{x} ; S \text { end }\{q\}, \\
& p \rightarrow t \geq 0 \\
& \{p\} P(\bar{x})\{q\}
\end{aligned}
$$

where $D=P(\bar{u}):: S, \operatorname{var}(\bar{x}) \cap \operatorname{var}(D)=\emptyset$ and $z$ is an integer variable that does not occur in $p, t, q$ and $S$ and is treated in the proof as a constant.

\section{Modularity}

Proof system $T R$ allows us to establish total correctness of recursive programs directly. However, sometimes it is more convenient to decompose the proof of total correctness into two separate proofs, one of partial correctness and one of termination. More 
specifically, given a correctness formula $\{p\} S\{q\}$, we first establish its partial correctness, using proof system $P R$. Then, to show termination it suffices to prove the simpler correctness formula $\{p\} S$ true $\}$ using proof system $T R$.

These two different proofs can be combined into one using the following general proof rule for total correctness:

\section{DECOMPOSITION}

$$
\begin{aligned}
& \vdash_{P R}\{p\} S\{q\}, \\
& \frac{\vdash_{T R}\{p\} S\{\text { true }\}}{\{p\} S\{q\}}
\end{aligned}
$$

where $\vdash_{P R}$ and $\vdash_{P R}$ refer to the proofs in the proof systems $P R$ and $T R$, respectively.

The decomposition rule and other auxiliary rules like A2 or A3 allow us to combine two correctness formulas derived independently. In some situations it is helpful to reason about procedure calls in a hierarchical way, by first deriving one correctness formula and then using it in a proof of another correctness formula. The following modification of the above simplified version of the recursion rule illustrates this principle, where we limit ourselves to a two-stage proof and one procedure:

\section{MODULARITY}

$$
\begin{aligned}
& \left\{p_{0}\right\} P(\bar{x})\left\{q_{0}\right\} \vdash\left\{p_{0}\right\} \text { begin local } \bar{u}:=\bar{x} ; S \text { end }\left\{q_{0}\right\}, \\
& \left\{p_{0}\right\} P(\bar{x})\left\{q_{0}\right\},\{p\} P(\bar{x})\{q\} \vdash\{p\} \text { begin local } \bar{u}:=\bar{x} ; S \text { end }\{q\} \\
& \{p\} P(\bar{x})\{q\}
\end{aligned}
$$

where $D=P(\bar{u}):: S$ and $\operatorname{var}(\bar{x}) \cap \operatorname{var}(D)=\emptyset$.

So first we derive an auxiliary property, $\left\{p_{0}\right\} P(\bar{x})\left\{q_{0}\right\}$ that we subsequently use in the proof of the 'main' property, $\{p\} P(\bar{x})\{q\}$. In general, more procedures may be used and an arbitrary 'chain' of auxiliary properties may be constructed. In the next section we show that such a modular approach can lead to better structured correctness proofs.

\section{Correctness Proof of the Quicksort Procedure}

We now apply the modular proof method to verify total correctness of the Quicksort algorithm, originally introduced in [10]. For a given array $a$ of type integer $\rightarrow$ integer and integers $x$ and $y$ this algorithm sorts the section $a[x: y]$ consisting of all elements $a[i]$ with $x \leq i \leq y$. Sorting is accomplished 'in situ', i.e., the elements of the initial (unsorted) array section are permuted to achieve the sorting property. We consider here the following version of Quicksort close to the one studied in [7]. It consists of a recursive procedure Quicksort $(m, n)$, where the formal parameters $m, n$ and the local variables $v, w$ are all of type integer: 


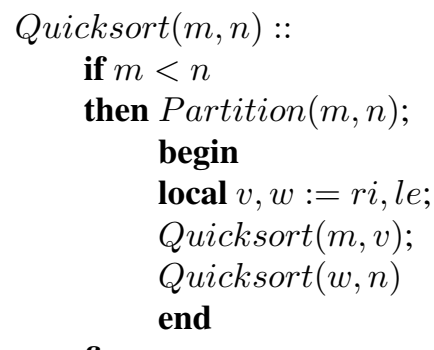

fi

Quicksort calls a non-recursive procedure Partition $(m, n)$ which partitions the array $a$ suitably, using global variables $r i, l e, p i$ of type integer standing for pivot, left, and right elements:

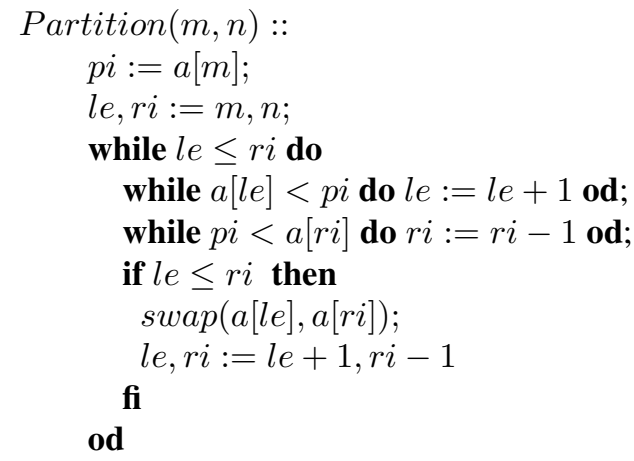

Here for two given simple or subscripted variables $u$ and $v$ the program $\operatorname{swap}(u, v)$ is used to swap the values of $u$ and $v$. So we stipulate that the following correctness formula

$$
\{x=u \wedge y=v\} \operatorname{swap}(u, v)\{x=v \wedge y=u\}
$$

holds in the sense of partial and total correctness, where $x$ and $y$ are fresh variables.

In the following $D$ denotes the set of the above two procedure declarations and $S_{Q}$ the body of the procedure Quicksort $(m, n)$.

\section{Formal Problem Specification}

Correctness of Quicksort amounts to proving that upon termination of the procedure call Quicksort $(m, n)$ the array section $a[m: n]$ is sorted and is a permutation of the input section. To write the desired correctness formula we introduce some notation. The assertion

$$
\operatorname{sorted}(a[x: y]) \equiv \forall i, j:(x \leq i \leq j \leq y \rightarrow a[i] \leq a[j])
$$

states that the integer array section $a[x: y]$ is sorted. To express the permutation property we use an auxiliary array $a_{0}$ in the section $a_{0}[x: y]$ of which we record the initial values of $a[x: y]$. The abbreviation

$$
\operatorname{bij}(f, x, y) \equiv f \text { is a bijection on } \mathbb{Z} \wedge \forall i \notin[x: y]: f(i)=i
$$

states that $f$ is a bijection on $\mathbb{Z}$ which is the identity outside the interval $[x: y]$. Hence 


$$
\operatorname{perm}\left(a, a_{0},[x: y]\right) \equiv \exists f:\left(b i j(f, x, y) \wedge \forall i: a[i]=a_{0}[f(i)]\right)
$$

specifies that the array section $a[x: y]$ is a permutation of the array section $a_{0}[x: y]$ and that $a$ and $a_{0}$ are the same elsewhere.

We can now express the correctness of Quicksort by means of the following correctness formula:

Q1 $\left\{a=a_{0}\right\}$ Quicksort $(x, y)\left\{\operatorname{perm}\left(a, a_{0},[x: y]\right) \wedge \operatorname{sorted}(a[x: y])\right\}$.

To prove correctness of Quicksort in the sense of partial correctness we proceed in stages and follow the methodology explained in Section 4 In other words, we establish some auxiliary correctness formulas first, using among others the recursion rule. Then we use them as premises in order to derive other correctness formulas, also using the recursion rule.

\section{Properties of Partition}

In the proofs we shall use a number of properties of the Partition procedure. This procedure is non-recursive, so to verify them it suffices to prove the corresponding properties of the procedure body using the proof systems $P D$ and $T D$, a task we leave to Nissim Francez.

More precisly, we assume the following properties of Partition in the sense of partial correctness:

$$
\begin{aligned}
& \text { P1 }\{\text { true }\} \operatorname{Partition}(m, n)\{r i \leq n \wedge m \leq l e\} \text {, } \\
& \text { P2 }\left\{x^{\prime} \leq m \wedge n \leq y^{\prime} \wedge \operatorname{perm}\left(a, a_{0},\left[x^{\prime}: y^{\prime}\right]\right)\right\} \\
& \text { Partition }(m, n) \\
& \left\{x^{\prime} \leq m \wedge n \leq y^{\prime} \wedge \operatorname{perm}\left(a, a_{0},\left[x^{\prime}: y^{\prime}\right]\right)\right\},
\end{aligned}
$$

\section{P3 true $\}$}

$$
\begin{aligned}
& \text { Partition }(m, n) \\
& \{l e>r i \wedge \\
& (\forall i \in[m: r i]: a[i] \leq p i) \wedge \\
& (\forall i \in[r i+1: l e-1]: a[i]=p i) \wedge \\
& (\forall i \in[l e: n]: p i \leq a[i])\},
\end{aligned}
$$

and the following property in the sense of total correctness:

$$
\text { P4 } \begin{aligned}
& \{m<n\} \\
& \text { Partition }(m, n) \\
& \{r i-m<n-m \wedge n-l e<n-m\} .
\end{aligned}
$$

Property $\mathbf{P 1}$ states the bounds for $r i$ and $l e$. We remark that $l e \leq n$ and $m \leq r i$ need not hold upon termination. Property $\mathbf{P 2}$ implies that the call Partition $(n, k)$ permutes the array section $a[m: n]$ and leaves other elements of $a$ intact, but actually is a stronger 
statement involving an interval $\left[x^{\prime}: y^{\prime}\right]$ that includes $[m: n]$, so that we can carry out the reasoning about the recursive calls of Quicksort. Finally, property $\mathbf{P 3}$ captures the main effect of the call Partition $(n, k)$ : the elements of the section $a[m: n]$ are rearranged into three parts, those smaller than $p i$ (namely $a[m: r i]$ ), those equal to $p i$ (namely $a[r i+1: l e-1]$ ), and those larger than $p i$ (namely $a[l e: n]$ ). Property $\mathbf{P 4}$ is needed in the termination proof of the Quicksort procedure: it states that the subsections $a[m: r i]$ and $a[l e: n]$ are strictly smaller than the section $a[m: n]$.

\section{Auxiliary proof: permutation property}

In the remainder of this section we use the following abbreviation:

$$
J \equiv m=x \wedge n=y .
$$

We first extend the permutation property $\mathbf{P 2}$ to the procedure Quicksort:

$$
\text { Q2 } \begin{aligned}
& \left\{\operatorname{perm}\left(a, a_{0},\left[x^{\prime}: y^{\prime}\right]\right) \wedge x^{\prime} \leq x \wedge y \leq y^{\prime}\right\} \\
& \text { Quicksort }(x, y) \\
& \left\{\operatorname{perm}\left(a, a_{0},\left[x^{\prime}: y^{\prime}\right]\right)\right\}
\end{aligned}
$$

Until further notice the provability symbol $\vdash$ refers to the proof system $P D$ augmented with the the block rule, the instantiation rule and the auxiliary rules $\mathrm{A} 2 \mathrm{A6}$

The appropriate claim needed for the application of the recursion rule is:

\section{Claim 1}

$$
\begin{aligned}
\mathbf{P 1}, \mathbf{P 2}, \mathbf{Q 2} \vdash & \left\{\operatorname{perm}\left(a, a_{0},\left[x^{\prime}: y^{\prime}\right]\right) \wedge x^{\prime} \leq x<y \leq y^{\prime}\right\} \\
& \text { begin local } m, n:=x, y ; S_{Q} \text { end } \\
& \left\{\operatorname{perm}\left(a, a_{0},\left[x^{\prime}: y^{\prime}\right]\right)\right\} .
\end{aligned}
$$

Proof. In Figure 1 a proof outline is given that uses as assumptions the correctness formulas P1, P2, and Q2. More specifically, the used correctness formula about the call of Partition is derived from $\mathbf{P 1}$ and $\mathbf{P 2}$ by the conjunction rule. In turn, the correctness formulas about the recursive calls of Quicksort are derived from $\mathbf{Q} 2$ by an application of the instantiation rule and the invariance rule. This concludes the proof of Claim 1.

We can now derive $\mathbf{Q 2}$ by the recursion rule. In summary, we proved

$$
\mathbf{P 1}, \mathbf{P 2} \vdash \mathbf{Q} 2 .
$$

\section{Auxiliary proof: sorting property}

We can now verify that the call Quicksort $(x, y)$ sorts the array section $a[x: y]$, so

Q3 $\{$ true $\}$ Quicksort $(x, y)\{\operatorname{sorted}(a[x: y])\}$.

The appropriate claim needed for the application of the recursion rule is:

\section{Claim 2}

P3, Q2, Q3 $\vdash\{$ true $\}$ begin local $m, n:=x, y ; S_{Q}$ end $\{\operatorname{sorted}(a[x: y])\}$. 


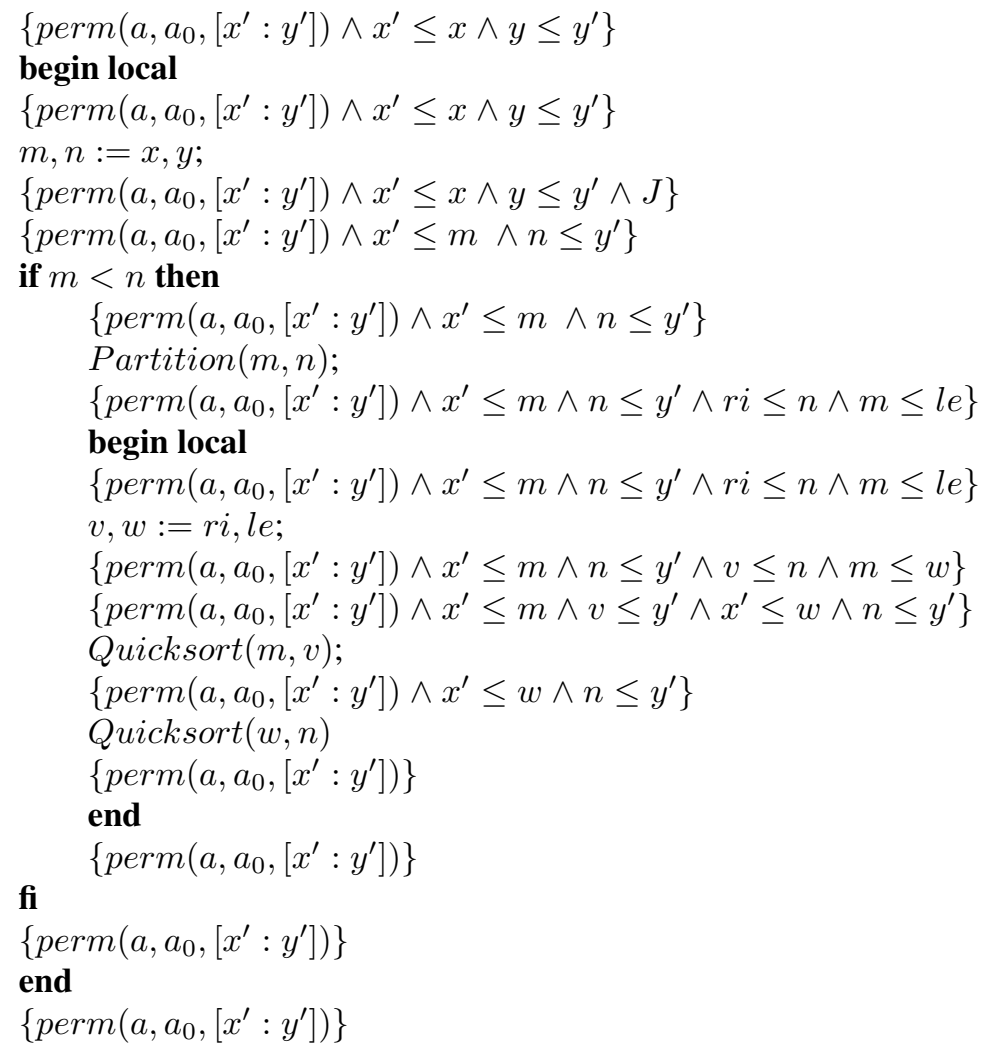

Fig. 1. Proof outline showing permutation property $\mathbf{Q 2}$

Proof. In Figure 2 a proof outline is given that uses as assumptions the correctness formulas P3, Q2, and Q3. In the following we justify the correctness formulas about Partition and the recursive calls of Quicksort used in this proof outline. In the postcondition of Partition we use the following abbreviation:

$$
\begin{aligned}
K \equiv & v<w \wedge \\
& (\forall i \in[m: v]: a[i] \leq p i) \wedge \\
& (\forall i \in[v+1: w-1]: a[i]=p i) \wedge \\
& (\forall i \in[w: n]: p i \leq a[i]) .
\end{aligned}
$$

Observe that the correctness formula

$$
\{J\} \operatorname{Partition}(m, n)\{J \wedge K[v, w:=r i, l e]\}
$$

is derived from $\mathbf{P 3}$ by the invariance rule. Next we verify the correctness formulas

$$
\{J \wedge K\} Q \text { uicksort }(m, v)\{\operatorname{sorted}(a[m: v]) \wedge J \wedge K\}
$$




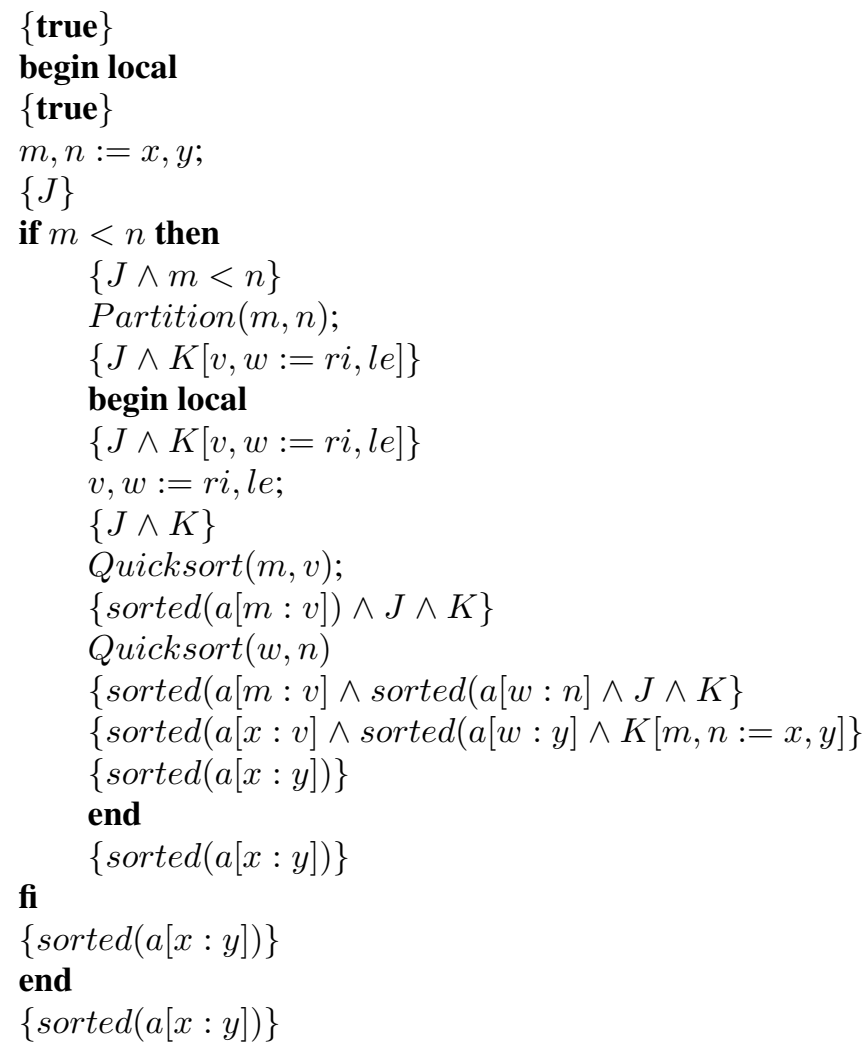

Fig. 2. Proof outline showing sorting property $\mathbf{Q 3}$

and

$$
\begin{aligned}
& \{\operatorname{sorted}(a[m: v]) \wedge J \wedge K\} \\
& \text { Quicksort }(w, n) \\
& \{\operatorname{sorted}(a[m: v] \wedge \operatorname{sorted}(a[w: n] \wedge J \wedge K\} .
\end{aligned}
$$

about the recursive calls of Quicksort.

Proof of (1). By applying the instantiation rule to Q3, we obtain

A1 $\{$ true $\}$ Quicksort $(m, v)\{\operatorname{sorted}(a[m: v])\}$.

Moreover, by the invariance axiom, we have

A2 $\{J\}$ Quicksort $(m, v)\{J\}$.

By applying the instantiation rule to $\mathbf{Q 2}$, we then obtain

$$
\begin{aligned}
& \left\{\operatorname{perm}\left(a, a_{0},\left[x^{\prime}: y^{\prime}\right]\right) \wedge x^{\prime} \leq m \wedge v \leq y^{\prime}\right\} \\
& \text { Quicksort }(m, v) \\
& \left\{\operatorname{perm}\left(a, a_{0},\left[x^{\prime}: y^{\prime}\right]\right)\right\} .
\end{aligned}
$$


Applying next the substitution rule with the substitution $\left[x^{\prime}, y^{\prime}:=m, v\right]$ yields

$$
\begin{aligned}
& \left\{\operatorname{perm}\left(a, a_{0},[m: v]\right) \wedge m \leq m \wedge v \leq v\right\} \\
& \operatorname{Quicksort}(m, v) \\
& \left\{\operatorname{perm}\left(a, a_{0},[m: v]\right)\right\} .
\end{aligned}
$$

So by a trivial application of the consequence rule, we obtain

$$
\left\{a=a_{0}\right\} \text { Quicksort }(m, v)\left\{\operatorname{perm}\left(a, a_{0},[m: v]\right)\right\} .
$$

We then obtain by an application of the invariance rule

$$
\left\{a=a_{0} \wedge K\left[a:=a_{0}\right]\right\} \text { Quicksort }(m, v)\left\{\operatorname{perm}\left(a, a_{0},[m: v]\right) \wedge K\left[a:=a_{0}\right]\right\} .
$$

Note now the following implications:

$$
\begin{aligned}
& K \rightarrow \exists a_{0}:\left(a=a_{0} \wedge K\left[a:=a_{0}\right]\right), \\
& \operatorname{perm}\left(a, a_{0},[m: v]\right) \wedge K\left[a:=a_{0}\right] \rightarrow K .
\end{aligned}
$$

So we conclude

A3 $\{K\}$ Quicksort $(m, v)\{K\}$

by the $\exists$-introduction rule and the consequence rule. Combining the correctness formulas A1-A3 by the conjunction rule we get (1).

Proof of (2). In a similar way as above, we can prove the correctness formula

$$
\left\{a=a_{0}\right\} \text { Quicksort }(w, n)\left\{p \operatorname{perm}\left(a, a_{0},[w: n]\right)\right\} .
$$

By an application of the invariance rule we obtain

$$
\begin{aligned}
& \left\{a=a_{0} \wedge \operatorname{sorted}\left(a_{0}[m: v]\right) \wedge v<w\right\} \\
& \text { Quicksort }(w, n) \\
& \left\{\operatorname{perm}\left(a, a_{0},[w: n]\right) \wedge \operatorname{sorted}\left(a_{0}[m: v]\right) \wedge v<w\right\}
\end{aligned}
$$

Note now the following implications:

$$
\begin{aligned}
& v<w \wedge \operatorname{sorted}(a[m: v]) \rightarrow \exists a_{0}:\left(a=a_{0} \wedge \operatorname{sorted}\left(a_{0}[m: v]\right) \wedge v<w\right), \\
& \left(\operatorname{perm}\left(a, a_{0},[w: n]\right) \wedge \operatorname{sorted}\left(a_{0}[m: v]\right) \wedge v<w\right) \rightarrow \operatorname{sorted}(a[m: v]) .
\end{aligned}
$$

So we conclude

B1 $\{v<w \wedge \operatorname{sorted}(a[m: v])\} \operatorname{Quicksort}(w, n)\{\operatorname{sorted}(a[m: v])\}$

by the $\exists$-introduction rule and the consequence rule. Further, by applying the instantiation rule to $\mathbf{Q 3}$ we obtain

B2 $\{$ true $\}$ Quicksort $(w, n)\{\operatorname{sorted}(a[w: n])\}$.

Next, by the invariance axiom we obtain 
B3 $\{J\}$ Quicksort $(w, m)\{J\}$.

Further, using the implications

$$
\begin{aligned}
& K \rightarrow \exists a_{0}:\left(a=a_{0} \wedge K\left[a:=a_{0}\right]\right), \\
& \operatorname{perm}\left(a, a_{0},[w: n]\right) \wedge K\left[a:=a_{0}\right] \rightarrow K,
\end{aligned}
$$

we can derive from $\mathbf{Q 2}$, in a similar manner as in the proof of $\mathbf{A 3}$,

B4 $\{K\}$ Quicksort $(w, n)\{K\}$.

Combining the correctness formulas B1-B4 by the conjunction rule and observing that $K \rightarrow v<w$ holds, we get (2).

The final application of the consequence rule in the proof outline given in Figure 2 is justified by the following crucial implication:

$$
\begin{aligned}
& \operatorname{sorted}(a[x: v]) \wedge \operatorname{sorted}(a[w: y]) \wedge K[m, n:=x, y] \rightarrow \\
& \operatorname{sorted}(a[x: y]) .
\end{aligned}
$$

Also note that $J \wedge m \geq n \rightarrow \operatorname{sorted}(a[x: y])$, so the implicit else branch is properly taken care of. This concludes the proof of Claim 2.

We can now derive $\mathbf{Q 3}$ by the recursion rule. In summary, we proved

$$
\mathbf{P 3}, \mathbf{Q 2} \vdash \mathbf{Q 3} .
$$

The proof of partial correctness of Quicksort is now immediate: it suffices to combine $\mathbf{Q 2}$ and $\mathbf{Q 3}$ by the conjunction rule. Then after applying the substitution rule with the substitution $\left[x^{\prime}, y^{\prime}:=x, y\right]$ and the consequence rule we obtain $\mathbf{Q 1}$, or more precisely

$$
\mathbf{P 1}, \mathbf{P 2}, \mathbf{P 3} \vdash \mathbf{Q 1} \text {. }
$$

\section{Total Correctness}

To prove termination, by the decomposition rule discussed in Section 4, it suffices to establish

Q4 $\{$ true $\}$ Quicksort $(x, y)\{$ true $\}$

in the sense of total correctness. In the proof we rely on the property $\mathbf{P 4}$ of Partition:

$$
\{m<n\} \operatorname{Partition}(m, n)\{r i-m<n-m \wedge n-l e<n-m\} .
$$

The provability symbol $\vdash$ refers below to the proof system $T D$ augmented with the block rule, the instantiation rule and the the auxiliary rules $\mathrm{A} 2 \mathrm{A6}$. For the termination proof of the recursive procedure call Quicksort $(x, y)$ we use

$$
t \equiv \max (y-x, 0)
$$


as the bound function. Since $t \geq 0$ holds, the appropriate claim needed for the application of the recursion rule II is:

\section{Claim 3}

$$
\begin{aligned}
& \text { P4, }\{t<z\} \text { Quicksort }(x, y)\{\text { true }\} \vdash \\
& \{t=z\} \text { begin local } m, n:=x, y ; S_{Q} \text { end }\{\text { true }\}
\end{aligned}
$$

Proof. In Figure 3 a proof outline for total correctness is given that uses as assumptions the correctness formulas $\mathbf{P 4}$ and $\{t<z\}$ Quicksort $(x, y)\{$ true $\}$. In the following we

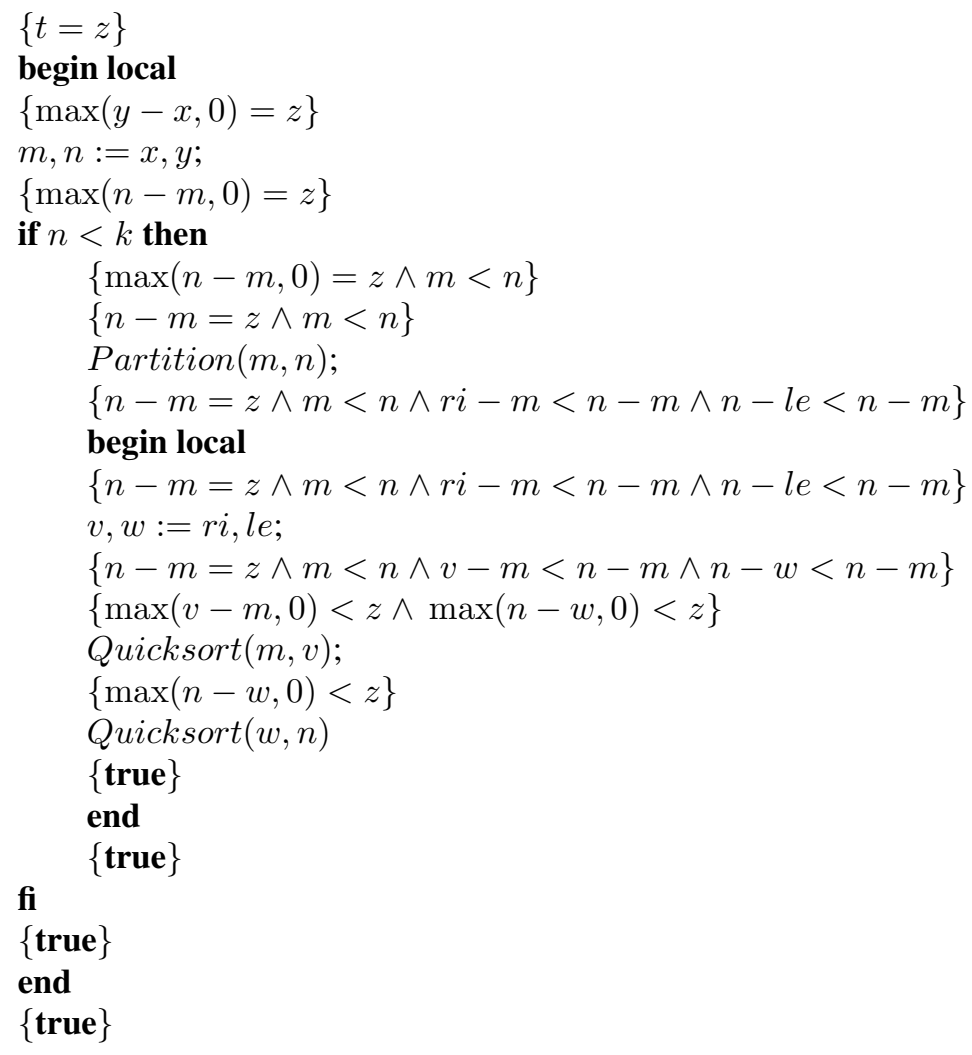

Fig. 3. Proof outline establishing termination of the Quicksort procedure

justify the correctness formulas about Partition and the recursive calls of Quicksort used in this proof outline. Since $m, n, z \notin$ change $(D)$, we deduce from P4 using the invariance rule the correctness formula

$$
\begin{aligned}
& \{n-m=z \wedge m<n\} \\
& \operatorname{Partition}(m, n) \\
& \{n-m=z \wedge r i-m<n-m \wedge n-l e<n-m\} .
\end{aligned}
$$


Consider now the assumption

$$
\{t<z\} \text { Quicksort }(x, y)\{\text { true }\} .
$$

Since $n, w, z \notin$ change $(D)$, the instantiation rule and the invariance rule yield

$$
\begin{aligned}
& \{\max (v-m, 0)<z \wedge \max (n-w, 0)<z\} \\
& \text { Quicksort }(m, v) \\
& \{\max (n-w, 0)<z\}
\end{aligned}
$$

and

$$
\{\max (n-w, 0)<z\} \text { Quicksort }(w, n)\{\text { true }\} .
$$

The application of the consequence rule preceding the first recursive call of Quicksort is justified by the following two implications:

$$
\begin{aligned}
& (n-m=z \wedge m<n \wedge v-m<n-m) \rightarrow \max (v-m, 0)<z, \\
& (n-m=z \wedge m<n \wedge n-w<n-m) \rightarrow \max (n-w, 0)<z .
\end{aligned}
$$

This completes the proof of Claim 3.

Applying now the simplified version of the recursion rule II we derive Q4. In summary, we proved

$$
\mathbf{P 4} \vdash \mathbf{Q 4} \text {. }
$$

\section{Conclusions}

The issue of modularity has been by now well-understood in the area of program construction. It also has been addressed in the program verification. Let us just mention two references, an early one and a recent one: [8] focused on modular verification of temporal properties of concurrent programs which were modelled as a set of modules that interact by means of procedure calls. In turn, [19] considered modular verification of heap manipulating programs, where the focus has been on the automatic extraction and verification specifications.

However, to our knowledge no approach has been proposed to deal with correctness of recursive programs in a modular fashion. When proving correctness of the Quicksort program we found that the simple approach here proposed allowed us to structure the proof better by establishing the 'permutation property' first and then using it in the proof of the 'sorting property'.

So in our approach we propose modularity at the level of proofs and not at the level of programs. This should be of help when organizing a mechanically verified correctness proof, by expressing the proofs of the subsidiary properties as subsidiary lemmas. In general, modular correctness proofs of programs are proofs from assumptions about subprograms, which can be considered as 'black boxes' of the given programs. Zwiers [20] has investigated an appropriate notion of completeness for such proofs from assumptions about black boxes, called modular completeness.

The first proof of partial correctness of Quicksort is given in [7]. That proof establishes the permutation and the sorting property simultaneously, in contrast to our 
approach. For dealing with recursive procedures, [7] use proof rules corresponding to our rules for blocks, instantiation, and recursion (for the case of one recursive procedure). They also use a so-called adaptation rule of [11] that allows one to adapt a given correctness formula about a program to other pre- and postconditions. In our approach we use several auxiliary rules which together have the same effect as the adaptation rule. The expressive power of the adaptation rule has been analyzed in [14]. No proof rule for the termination of recursive procedures is proposed in [7], only an informal argument is given why Quicksort terminates. An informal proof of total correctness of Partition is given in [12] as part of the program Find given in [9].

The recursion rule is modelled after the so-called Scott induction rule for fixed points that appeared first in the unpublished manuscript Scott and De Bakker [18]. Recursion rule II for total correctness is taken from America and De Boer [1], where also the completeness of a proof system similar to $T R$ is established. The modularity rule corresponds to a theorem due to Bekić [5] which states that for systems of monotonic functions iterative fixed points coincide with simultaneous fixed points.

\section{Acknowledgment}

We thank the reviewer for helpful suggestions.

\section{References}

1. America, P., de Boer, F.S.: Proving total correctness of recursive procedures. Information and Computation 84(2), 129-162 (1990)

2. Apt, K.R.: Ten years of Hoare's logic, a survey, part I. ACM Transactions on Programming Languages and Systems 3, 431-483 (1981)

3. Apt, K.R., de Boer, F.S., Olderog, E.-R.: Verification of Sequential and Concurrent Programs, 3rd extended edn. Springer, New York (2009) (to appear)

4. Barendregt, H.P.: The Lambda Calculus. North Holland, Amsterdam (1984)

5. Bekić, H.: Definable operations in general algebras, and the theory of automata and flow charts. Technical report, IBM Laboratory, Vienna (1969); Typescript

6. Filliâtre, J.-C., Magaud, N.: Certification of sorting algorithms in the system Coq. In: Theorem Proving in Higher Order Logics: Emerging Trends (1999)

7. Foley, M., Hoare, C.A.R.: Proof of a recursive program: Quicksort. Computer Journal 14(4), 391-395 (1971)

8. Hailpern, B., Owicki, S.: Modular verification of concurrent programs. In: POPL 1982: Proceedings of the 9th ACM SIGPLAN-SIGACT symposium on Principles of programming languages, pp. 322-336. ACM, New York (1982)

9. Hoare, C.A.R.: Algorithm 65, Find. Communications of the ACM 4(7), 321 (1961)

10. Hoare, C.A.R.: Quicksort. Comput. J. 5(1), 10-15 (1962)

11. Hoare, C.A.R.: Procedures and parameters: an axiomatic approach. In: Engeler, E. (ed.) Proceedings of Symposium on the Semantics of Algorithmic Languages, New York. Lecture Notes in Mathematics, vol. 188, pp. 102-116. Springer, Heidelberg (1971)

12. Hoare, C.A.R.: Proof of a program: Find. Communications of the ACM 14(1), 39-45 (1971)

13. Kaldewaij, A.: Programming: The Derivation of Algorithms. Prentice-Hall, Englewood Cliffs (1990) 
14. Olderog, E.-R.: On the notion of expressiveness and the rule of adaptation. Theoretical Computer Science 30, 337-347 (1983)

15. Owre, S., Shankar, N.: Writing PVS proof strategies. In: Archer, M., Di Vito, B., Muñoz, C. (eds.) Design and Application of Strategies/Tactics in Higher Order Logics (STRATA 2003), number CP-2003-212448 in NASA Conference Publication, Hampton, VA, September 2003, pp. 1-15. NASA Langley Research Center (2003)

16. Plotkin, G.D.: A structural approach to operational semantics. Technical Report DAIMI-FN 19, Department of Computer Science, Aarhus University (1981)

17. Plotkin, G.D.: A structural approach to operational semantics. J. of Logic and Algebraic Programming, 60-61, 17-139 (2004); Revised version of [16]

18. Scott, D., de Bakker, J.W.: A theory of programs. Notes of an IBM Vienna Seminar (1969)

19. Taghdiri, M.: Automating Modular Program Verification by Refining Specifications. Ph.D thesis. MIT, Cambridge, Mass (2008), http://alloy.mit.edu/community/files/mana_thesis.pdf

20. Zwiers, J.: Compositionality, Concurrency, and Partial Correctness. LNCS, vol. 321. Springer, Heidelberg (1989)

\section{Appendix}

We list here the used axioms and proof rules that were not defined earlier in the text. To establish correctness of while programs we rely on the following axioms and proof rules. In the proofs of partial correctness the loop rule is used, while in the proofs of total correctness the loop II rule is used.

AXIOM 1: SKIP

$$
\{p\} \operatorname{skip}\{p\}
$$

AXIOM 2: ASSIGNMENT

$$
\{p[u:=t]\} u:=t\{p\}
$$

AXIOM 3: PARALLEL ASSIGNMENT

$$
\{p[\bar{x}:=\bar{t}]\} \bar{x}:=\bar{t}\{p\}
$$

RULE 4: COMPOSITION

$$
\frac{\{p\} S_{1}\{r\},\{r\} S_{2}\{q\}}{\{p\} S_{1} ; S_{2}\{q\}}
$$

RULE 5: CONDITIONAL

$$
\frac{\{p \wedge B\} S_{1}\{q\},\{p \wedge \neg B\} S_{2}\{q\}}{\{p\} \text { if } B \text { then } S_{1} \text { else } S_{2} \text { fi }\{q\}}
$$


RULE 6: LOOP

$$
\frac{\{p \wedge B\} S\{p\}}{\{p\} \text { while } B \text { do } S \operatorname{od}\{p \wedge \neg B\}}
$$

RULE 7: CONSEQUENCE

$$
\frac{p \rightarrow p_{1},\left\{p_{1}\right\} S\left\{q_{1}\right\}, q_{1} \rightarrow q}{\{p\} S\{q\}}
$$

RULE 8: LOOP II

$$
\begin{aligned}
& \{p \wedge B\} S\{p\}, \\
& \{p \wedge B \wedge t=z\} S\{t<z\} \\
& p \rightarrow t \geq 0 \\
& \{p\} \text { while } B \text { do } S \text { od }\{p \wedge \neg B\}
\end{aligned}
$$

where $t$ is an integer expression and $z$ is an integer variable that does not appear in $p, B, t$ or $S$.

Additionally, we rely on the following auxiliary axioms and proof rules that occasionally refer to the assumed set of procedure declarations $D$.

AXIOM A1: INVARIANCE

$$
\{p\} S\{p\}
$$

where $\operatorname{free}(p) \cap(\operatorname{change}(D) \cup \operatorname{change}(S))=\emptyset$.

RULE A2: DISJUNCTION

$$
\frac{\{p\} S\{q\},\{r\} S\{q\}}{\{p \vee r\} S\{q\}}
$$

RULE A3: CONJUNCTION

$$
\frac{\left\{p_{1}\right\} S\left\{q_{1}\right\},\left\{p_{2}\right\} S\left\{q_{2}\right\}}{\left\{p_{1} \wedge p_{2}\right\} S\left\{q_{1} \wedge q_{2}\right\}}
$$

RULE A4: $\exists$-INTRODUCTION

$$
\frac{\{p\} S\{q\}}{\{\exists x: p\} S\{q\}}
$$

where $x \notin$ change $(D) \cup$ change $(S) \cup \operatorname{free}(q)$.

RULE A5: INVARIANCE

$$
\frac{\{r\} S\{q\}}{\{p \wedge r\} S\{p \wedge q\}}
$$


where $\operatorname{free}(p) \cap($ change $(D) \cup$ change $(S))=\emptyset$.

\section{RULE A6: SUBSTITUTION}

$$
\frac{\{p\} S\{q\}}{\{p[\bar{z}:=\bar{t}]\} S\{q[\bar{z}:=\bar{t}]\}}
$$

where $(\operatorname{var}(\bar{z}) \cup \operatorname{var}(\bar{t})) \cap(\operatorname{change}(D) \cup \operatorname{change}(S))=\emptyset$. 